\title{
RelA/p65 promotes osteoclast differentiation by blocking a RANKL-induced apoptotic JNK pathway in mice
}

\author{
Sergio Vaira, ${ }^{1}$ Muhammad Alhawagri, ${ }^{1}$ Imani Anwisye, ${ }^{1}$ Hideki Kitaura, ${ }^{2}$ \\ Roberta Faccio, ${ }^{2}$ and Deborah Veis Novack ${ }^{1,3}$ \\ 1Division of Bone and Mineral Diseases, Department of Medicine, ${ }^{2}$ Department of Orthopedic Surgery, and \\ 3Department of Pathology, Washington University School of Medicine, St. Louis, Missouri, USA.
}

\begin{abstract}
Osteoclasts (OCs) function to reabsorb bone and are responsible for the bone loss associated with inflammatory arthritis and osteoporosis. OC numbers are elevated in most disorders of accelerated bone destruction, reflecting altered rates of precursor differentiation and apoptosis. Both of these processes are regulated by the JNK family of MAP kinases. In this study, we have demonstrated that the NF- $\kappa B$ subunit RelA/p65 inhibits JNKmediated apoptosis during a critical period of commitment to the $\mathrm{OC}$ phenotype in response to the cytokine RANKL. This RelA/p65-mediated arrest of cell death led to enhanced OC differentiation. Hence, Rela ${ }^{-/}$ OC precursors displayed prolonged JNK activation in response to RANKL, and this was accompanied by an increase in cell death that prevented efficient differentiation. Although complete blockade of JNK activity inhibits osteoclastogenesis, both short-term blockade in RelA-deficient cultures and suppression of the downstream mediator, Bid rescued apoptosis and differentiation. These antiapoptotic effects were RelA specific, as overexpression of RelA, but not RelB, blocked apoptosis and rescued differentiation in Rela ${ }^{-/-}$precursors. Thus, RelA blocks a RANKL-induced, apoptotic JNK-Bid pathway, thereby promoting OC differentiation. Consistent with this, mice lacking RelA/p65 in the hematopoietic compartment were shown to have a deficient osteoclastogenic response to RANKL and were protected from arthritis-induced osteolysis.
\end{abstract}

\section{Introduction}

Osteoclasts (OCs) are multinucleated cells derived from myeloid bone marrow progenitors that express RANK, the receptor for the key osteoclastogenic cytokine RANKL (1). Increased OC formation and activity is observed in many osteopenic disorders, including postmenopausal osteoporosis, lytic bone metastasis, and rheumatoid arthritis, and leads to pain and structural instability. Osteoblast lineage cells express a membrane-bound form of RANKL, a member of the TNF cytokine family. Like other members of the TNF receptor superfamily, RANK strongly activates the NF-кB pathway.

In mammals, the NF-кB family has 5 members: RelA/p65, RelB,

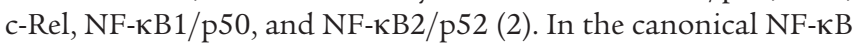
pathway, ligation of RANK activates the inhibitor of IкB kinase

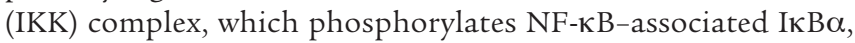
leading to its ubiquitination and proteosomal degradation. These events release NF- $\kappa$ B dimers containing RelA and c-Rel in the cytosol, allowing them to translocate into the nucleus where they enhance transcription of target genes. In the alternative NF- $\mathrm{BB}$ pathway, NF-KB-inducing kinase (NIK) and IKK $\alpha$ target p100 for ubiquitination and processing, thereby allowing nuclear translocation of predominantly RelB-containing dimers.

The importance of NF-кB in osteoclastogenesis has been high-

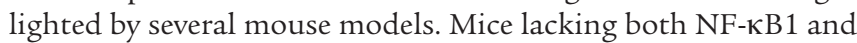
NF-кB2 have severe osteopetrosis with complete absence of OCs

Nonstandard abbreviations used: BMM, bone marrow macrophage; CTX, collagen C-terminal telopeptide; IKK, IKB kinase; MTT, 3-4, 5-dimethylthiazol-2y-2,5 diphenyltetrazolium bromide; NIK, NF-кB-inducing kinase; OC, osteoclast; RC, radiation chimera; TNFR1, p55 TNF receptor; TRAP, tartrate resistant acid phosphatase. Conflict of interest: The authors have declared that no conflict of interest exists. Citation for this article: J. Clin. Invest. 118:2088-2097 (2008). doi:10.1172/JCI33392.
$(3,4)$. Ablation of IKK $\beta$, a key component of the IKK complex, blocks nuclear translocation of RelA, RelB, and c-Rel and leads to increased bone mass and OC lineage apoptosis (5). Transduction of OC precursors with a superrepressor of IкB $\alpha$ that cannot be proteasomally degraded blocks OC differentiation and survival $(6,7)$. Additionally, we have previously shown that ablation of NIK inhibits both RelA and RelB nuclear translocation in pre-OCs, leading to a resistance to RANKL-induced osteoclastogenesis in vivo and in vitro (8). Consistent with this observation, osteoclastogenesis and hence bone erosion are reduced in the serum transfer model of inflammatory arthritis in $\mathrm{Nik}^{-/-}$mice (9). These data suggest that tight regulation of the classical and alternative pathways is critical to the OC in both physiological and pathological circumstances. However, none of the previous studies have addressed the role of individual subunits in the OC response to RANKL.

Ablation of RelA in mice causes embryonic lethality, with extensive liver apoptosis at E15.5 (10), dependent on TNF- $\alpha$. Thus, deficiency of either p55 TNF receptor (TNFR1) or TNF- $\alpha$ rescues this hepatic event and allows the doubly mutant mice to live at least to postnatal day $14-21(11,12)$. Ablation of TNFR1 in the IKK $\beta$ mutant mice also restored OC numbers in vivo, suggesting that TNF is the main driver of apoptosis in this lineage as well (5). One report has suggested that RANKL also induces OC lineage apoptosis, despite the lack of a death domain in its receptor RANK (13), although the mechanism has not been extensively explored.

Using genetic knockout approaches, the specific role of RelA has previously been examined in lymphocytes, lymph node stroma, hepatocytes, and neurons. Although several groups have demonstrated potent RelA activation in OCs in response to RANKL and/or TNF, no studies have specifically addressed the unique role of this subunit. We have now used Rela-/-Tnfr1 $1^{-/}$mice to explore the role 
A
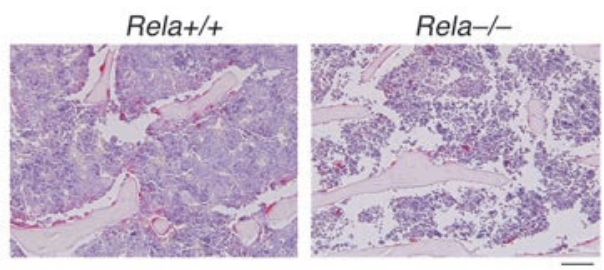

B
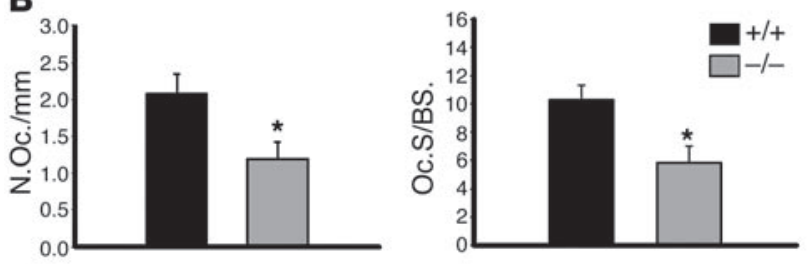

C
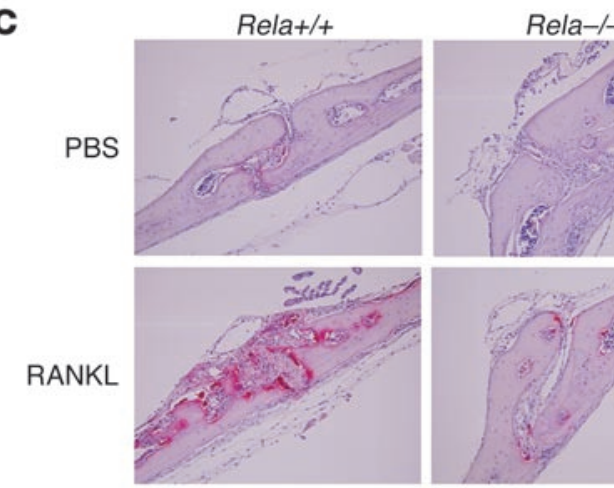

D

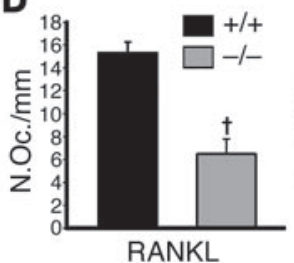

RANKL

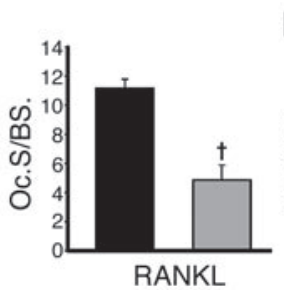

E

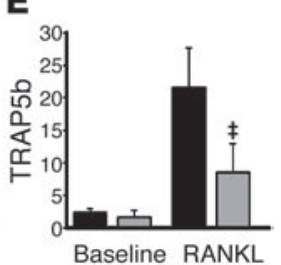

of RelA in the OC lineage and find that this subunit plays a critical role in opposing cell death induced by RANKL during OC differentiation. This apoptotic pathway is mediated by JNK and cannot be blocked by activation of RelB.

\section{Results}

Rela is important for RANKL-stimulated osteoclastogenesis in vivo. Although RelA-deficiency does not cause embryonic lethality on the Tnfr1 $1^{-/-}$background, the double-knockout mice live an average of only $2-3$ weeks. In order to analyze the subunit-specific role of RelA in the OCs of adult mice, we generated radiation chimeras (RCs), engrafting C57BL/6J mice with bone marrow cells from Rela-/-Tnfr1 $1^{-/-}$donors or Rela ${ }^{+/}{ }^{-} \mathrm{nfr}^{-1^{--}}$controls. Hematopoietic cells in the RCs are all Tnfr $1^{-/-}$regardless of Rela genotype, and we will subsequently refer to these RCs as either $\mathrm{Rela}^{+/+}$or Rela ${ }^{-/}$. We performed histomorphometric analysis on vertebrae from RCs 4 months after engraftment and found a $50 \%$ decrease in OC number and surface in Rela ${ }^{-/-}$relative to WT (Figure 1, A and B). There was no difference in trabecular bone volume (BV/TV) in Rela ${ }^{+/}$and Rela ${ }^{-/}$chimeras. However, others have failed to detect differences in BV/TV in RCs bearing marrow with strong OC phenotypes such as $R_{A N K^{-/}}$(J. Lorenzo, personal communication).

\section{Figure 1}

RelA is important for basal and stimulated OC formation in vivo. (A) TRAP-stained sections of vertebrae from $\mathrm{Rela}^{+/+}$and $\mathrm{Rela}^{-/-} \mathrm{RCs}$ 4 months after bone marrow reconstitution. Scale bar: $100 \mu \mathrm{m}$. (B) Histomorphometric analysis was performed on TRAP-stained sections to determine the OC number (N.Oc./mm) and OC surface (Oc.S./BS.). ${ }^{*} P<0.05 ; n=8$ /group. (C) Rela ${ }^{+/+}$and Rela ${ }^{-/-}$RCs were injected with 100- $\mu \mathrm{g}$ RANKL or PBS daily for 5 days and sacrificed on the sixth day. TRAP-stained coronal sections of calvaria at the sagittal suture show a consistent increase in OCs along sutures and sinusoids only in Rela+/+ RANKL-treated animals. Scale bar: $200 \mu \mathrm{m}$. (D) Quantification of the N.Oc./mm and Oc.S./BS. (percentage of bone surface covered by OCs) along calvarial surfaces of mice in $\mathbf{C}$ confirms the significant difference in response of $\mathrm{Rela}^{+/+}$and $\mathrm{Rela}^{-/-} \mathrm{RC}$ to RANKL injection. In the PBS-injected animals, only rare OCs are detected in either genotype. ${ }^{\dagger} P<0.005 ; n=5 /$ group. (E) Serum levels of TRAP5b were determined 1 day prior to first RANKL injection (baseline) and at sacrifice on day 6 (RANKL). ${ }^{\ddagger} P=0.01 ; n=5$ /group.

The key cytokine required for OC differentiation from monocyte lineage precursors is RANKL. To examine the osteoclastogenic response to RANKL more directly, we injected RANKL $(100 \mu \mathrm{g} / \mathrm{d}$, for 5 days subcutaneously) into Rela ${ }^{+/+}$and Rela ${ }^{-/}$RCs. Examination of tartrate resistant acid phosphatase-stained (TRAP) calvaria sections showed that the Rela ${ }^{+/+}$controls had an increased OC number along calvarial sinuses and sutures compared with the PBS-injected controls (Figure 1, C and D). In contrast, Rela ${ }^{-/}$RCs exhibited a blunted response to RANKL, indicated by fewer TRAP ${ }^{+}$ OCs on histomorphometric analysis and a significantly smaller rise in serum TRAP5b levels (Figure 1E). Thus, RelA is important for RANKL-stimulated osteoclastogenesis in vivo.

Absence of RelA protects mice from arthritis-induced bone loss but not inflammation. Blockade of NF-KB reduces both inflammation and bone loss in several murine models of inflammatory arthritis (9, 14, 15). The Rela ${ }^{+/}$and Rela $/-$RCs were injected with K/BxN serum (16) on days 0,2 , and 7 , with LPS on day 2 , to induce serum transfer arthritis. Histological sections of hind paws harvested at day 14 were stained with H\&E and TRAP. H\&E sections from Rela/++ and Relar/RCs exhibited the same rate of infiltrate in the joint space, composed primarily of neutrophils (Figure 2A), and the thickness of the hind paws of Rela $^{-1}$ RCs was very similar to those of $\mathrm{Rela}^{+/+}$controls through day 14 (Figure 2B), indicating that RelA is not important for the inflammatory response in this model. Another common feature of serum transfer arthritis is erosion of bones in the hind paw, with a large induction of osteoclastogenesis. Hind paws from Rela ${ }^{-1}$ RCs had fewer OCs compared with Relat/+ RCs, both on the bone surface and in adjacent marrow, with 4-fold fewer OCs on the external bone surfaces (Figure 2, C and D). These results correlated with blunted induction of the serum marker collagen C-terminal telopeptide (CTX) at day 14 in Rela-/- RCs relative to Rela $^{+/+}$(Figure 2E). These results suggest that the RelA subunit is required for the osteoclastogenic response to inflammation.

RelA is important for OC precursor survival but not differentiation. To better understand the mechanism by which RelA impacts the $\mathrm{OC}$, we turned to in vitro culture. In the presence of M-CSF and RANKL or in coculture with wild-type osteoblasts, bone marrow macrophages (BMMs) from RelA-deficient mice formed fewer OCs (Figure 3, A and B). The effect is dependent on the loss of RelA and not TNFR1, since reexpression of the latter did not rescue the phenotype (data not shown). During culture, we noticed fewer cells in RelA-deficient wells treated with RANKL and M-CSF, but 
A
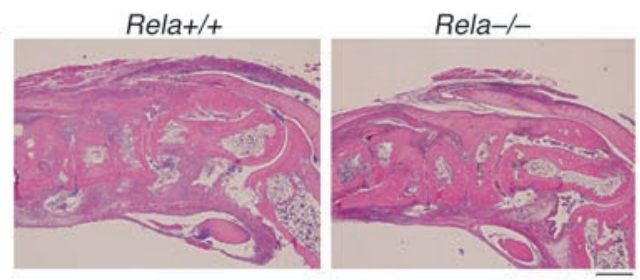

C

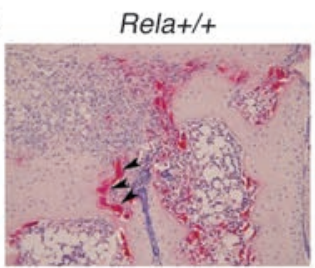

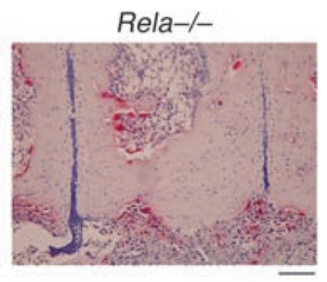

B

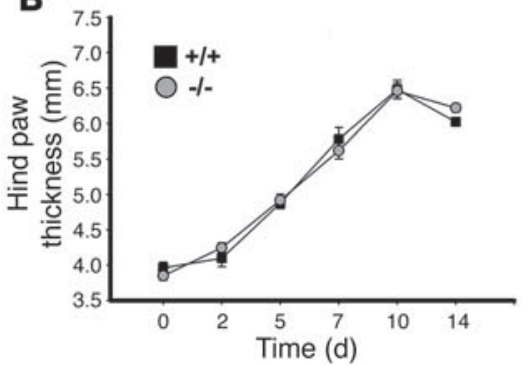

D

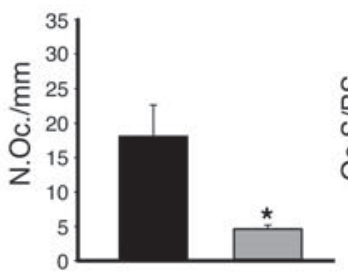

$\mathbf{E}$

\section{Figure 2}

Rela ${ }^{-/}$RCs are protected from arthritis-induced bone loss. (A) Arthritis was induced in Relat++ and Rela ${ }^{-/-}$RCs 5 weeks after marrow transplantation by injection of K/BxN serum at days 0,2 , and 7 , with LPS on day 2 . Histological sections of ankle joints at day 14 stained with H\&E show an extensive and similar inflammatory infiltrate in the joint spaces. Scale bar: $500 \mu \mathrm{m}$. (B) The inflammatory response was also evaluated by measurement of hind paw thickness (sum of both paws) during induction of arthritis. $n=8 /$ group. The experiment was repeated twice, with similar results. (C) TRAP-stained sections of metatarsals demonstrate OCs both on the outer bone surface adjacent to inflammatory pannus (arrowheads) and in marrow spaces. Scale bar: $100 \mu \mathrm{m}$. (D) Histomorphometric analysis of N.Oc./mm and Oc.S./BS. on the outer bone surface demonstrate significantly more OCs in Rela ${ }^{++}$RCs compared with Rela ${ }^{-/}$RCs. ${ }^{*} P<0.001 ; n=8 /$ group. (E) Serum CTX was measured at day 0 and 14 of serum transfer arthritis, and the fold change for each RC was plotted, demonstrating a significant rise only in the $\operatorname{Rela}^{+/+}$group. ${ }^{\dagger} P<0.05 ; n=6 /$ group.

not in RelA-deficient wells treated with M-CSF alone. MTT assays (3-4, 5-dimethylthiazol-2y-2,5 diphenyltetrazolium bromide assays) confirmed a decreased cell number in Rela ${ }^{-/}$cultures by 48 hours of RANKL treatment (Figure 3C), although there was no difference between Rela $^{+/+}$and Rela ${ }^{-/-}$cells cultured in M-CSF alone (data not shown). These data suggested that RelA plays an antiapoptotic role. Therefore, we examined various markers of apoptosis in RANKL-stimulated cultures. In concordance with the MTT assay showing that significant cell loss in Rela/- cultures occurs between 24 and 48 hours of RANKL treatment, a DNA fragmentation assay showed that Rela $^{-1}$ cultures treated with RANKL for 36 hours, but not 18 hours, had 3- to 4-fold higher levels of DNA fragmentation than M-CSF treated controls (Figure 3D). Interestingly, Rela ${ }^{+/+}$ cultures also had significant levels of DNA fragmentation after 36 hours of RANKL exposure, although the levels were much less than for similarly treated Rela ${ }^{-1}$. This RANKL-mediated apoptosis, accentuated by the absence of RelA, was confirmed by examination of caspase-3. Using a fluorimetric assay (Figure $3 \mathrm{E}$ ), we found that while caspase- 3 activation increased modestly (2-fold) in $\mathrm{Rela}^{+/+}$precursors between 36 and 48 hours of RANKL exposure, caspase- 3 was activated up to 4 -fold in similarly treated Rela ${ }^{-/-}$cells during the same period. Caspase- 9 activation was also elevated in the absence of RelA, while caspase- 8 levels were unaffected (data not shown). These data indicate that RelA protects OC precursors from RANKL-induced cell death by blocking an intrinsic (caspase$3 / 9$ dependent) apoptotic pathway during a critical period of differentiation (36-48 hours of RANKL exposure).

In order to determine if the failure to generate OCs was secondary to apoptosis and not due to a primary RelA effect on differentiation, we used the caspase inhibitor ZVAD, in conjunction with RANKL, during the first 48 hours of OC differentiation.
This treatment blocked the RANKL-induced caspase- 3 activation (Figure $3 \mathrm{E}$ ) and normalized the number of OCs generated from Rela $^{-/-}$precursors after 6 days of culture (Figure 3F). We also found that Rela-/- OCs generated in the presence of ZVAD were able to form resorptive pits on bone slices (Figure 3G). These results demonstrate that RelA is important for OC precursor survival but not differentiation or function.

A JNK-Bid pathway causes RANKL-mediated apoptosis in the absence of RelA. NF-кB has been implicated in the control of many antiapoptotic genes, including $B c l 2, B c l x L$, and the inhibitor of apoptosis (IAP) family (Ciap1, -2, Xiap). We therefore performed real-time quantitative PCR analysis to examine expression levels of these potential RelA targets in OC precursors. Of this group, only xiap was induced by RANKL in a RelA-dependent manner (Figure 4A, left panel). XIAP binds to and inhibits caspase-3 (17), and, therefore, may represent an important antiapoptotic signal controlled by RelA in response to RANKL in OC precursors.

In the context of TNF- $\alpha$-mediated apoptosis, absence of RelA potentiates JNK activation. One mechanism is decreased via expression of Gadd45 $\beta$, an inhibitor of the JNK kinase MKK7 (18). Others have described reduced activity of the MKP family of JNK phosphatases (19). In OC precursors, RANKL-induced expression of both Gadd45b and Mkp5 in a RelA-dependent manner (Figure $4 \mathrm{~A}$ ), suggesting that JNK activation might also be altered in Rela ${ }^{-/}$ OC precursors. Although RANKL-induced JNK activation was normal in Rela ${ }^{-/-}$BMMs (data not shown), Rela ${ }^{-/}$pre-OCs (BMMs cultured for 2 days in M-CSF and RANKL) showed stronger phosphorylation of JNK following starvation and restimulation with RANKL for 15 minutes (Figure 4B) and increased JNK kinase activity (data not shown). To determine whether this enhanced JNK activation is related to apoptosis in pre-OCs, we treated $\mathrm{Rela}^{-/}$cultures 
A

\section{A}

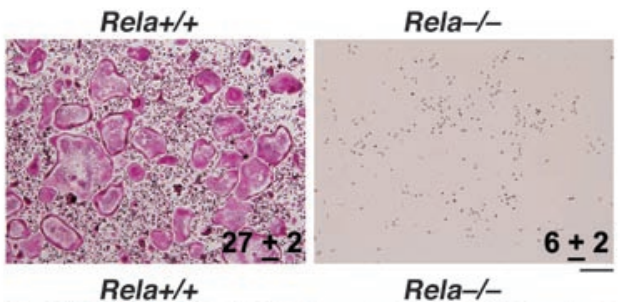

B

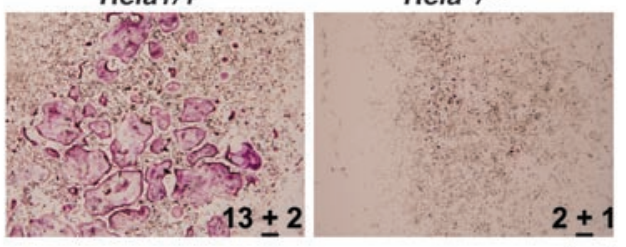

C

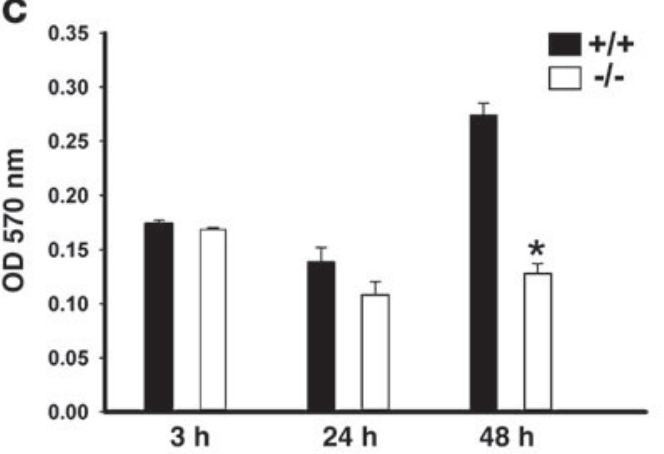

D

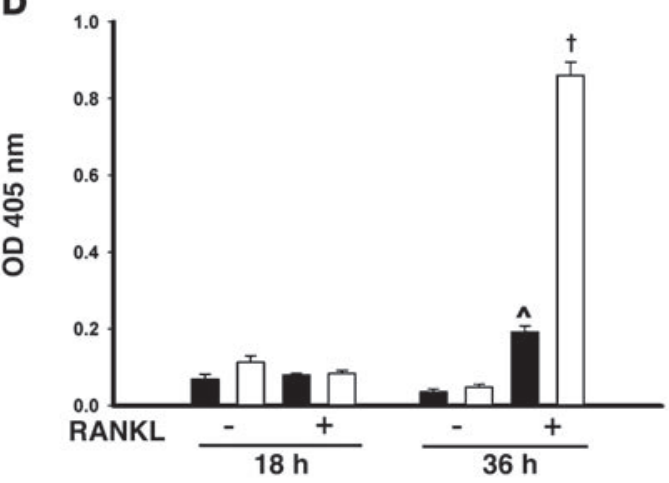

E

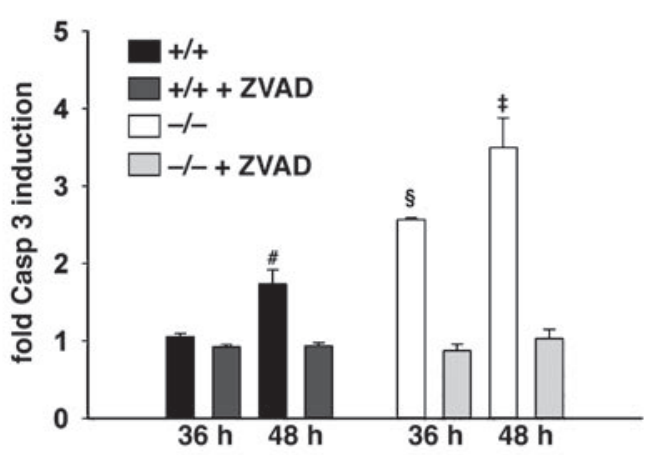

F

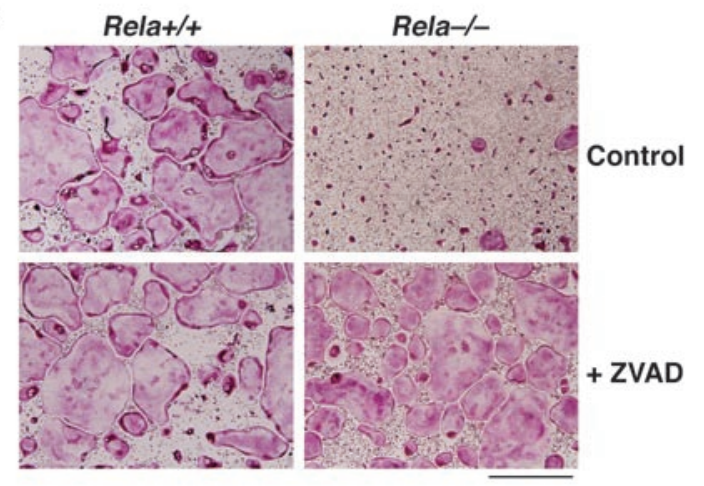

G
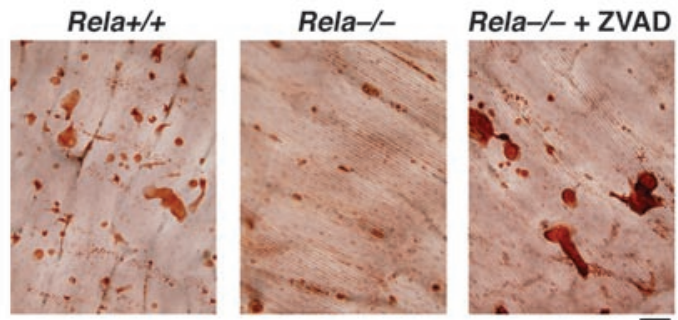

\section{Figure 3}

Rela-l- BMMs are sensitive to RANKL-induced cell death. (A) Rela ${ }^{+/+}$or Rela ${ }^{-/-}$BMMs were cultured in M-CSF and RANKL or (B) cultured with wild-type calvarial osteoblasts in the presence of 1,25 vitamin $D_{3}$, then fixed and TRAP stained. Scale bar: $500 \mu \mathrm{m}$. The number of OCs per field (mean \pm SEM) is shown, demonstrating fewer OCs in the absence of RelA. (C) Cell number was assessed by MTT assay in BMMs cultured with M-CSF and RANKL for 3-48 hours, showing fewer cells after 48 hours in Rela ${ }^{-/}$cultures relative to Rela ${ }^{+/+}\left({ }^{*} P<0.00001 ; n=4\right)$. (D) Apoptosis was measured by DNA fragmentation assay after 18 or 36 hours in the presence of M-CSF, with (+) or without (-) RANKL. After 36 hours, Rela-l- BMMs cultured with M-CSF and RANKL, but not with M-CSF alone, had increased cell death. ${ }^{\wedge} P<0.0001$ compared with Relat+/ without RANKL at 36 hours; $+P<0.00001$ relative to Rela ${ }^{+/+}$with RANKL at 36 hours. (E) Caspase-3 (casp 3) activation was measured in Rela ${ }^{+/+}$and Rela-1-BMMs cultured in M-CSF/RANKL for 36 and 48 hours, with or without ZVAD, and fold induction relative to the same cells in M-CSF alone was plotted. ${ }^{\prime} P<0.05$ relative to Rela ${ }^{+/+} 36$ hours; $¥ P<0.01, \$ P<0.00001$ relative to Rela ${ }^{+/+}$at 36 or 48 hours. $(F)$ OCs were generated with or without ZVAD during the first 48 hours. Scale bar: $500 \mu \mathrm{m}$. (G) OCs were generated as in F on cortical bone slices. Resorption pits (brown) were stained with horseradish peroxidase-conjugated wheat germ agglutinin. Scale bar: $100 \mu \mathrm{m}$.

with the JNK inhibitor SP600125, along with RANKL for 36 hours. Inhibition of JNK reduced DNA fragmentation to wild-type levels (Figure 4C) and restored osteoclastogenesis in Rela ${ }^{-1}$ precursors (Figure 4D), suggesting that JNK is mechanistically involved in RANKL-induced apoptosis in RelA-deficient OC precursors.

Enhanced JNK activation appears to initiate apoptosis by activating $\mathrm{Bid}$, a proapoptotic $\mathrm{BH} 3$-only member of $\mathrm{Bcl}-2$ family, at least in response to TNF (20). While Bid was expressed in both Relat++ and Rela ${ }^{-/}$BMM cultures, levels were not affected by RANKL treatment in either genotype (Figure 5A), and cleavage products were not detected. In order to determine whether Bid is responsible for RANKL-induced apoptosis, we employed a retroviral siRNA approach, using 2 different sequences to knock down Bid expression in Rela ${ }^{-/}$OC precursors. Levels of Bid in Rela ${ }^{-/-}$BMMs 


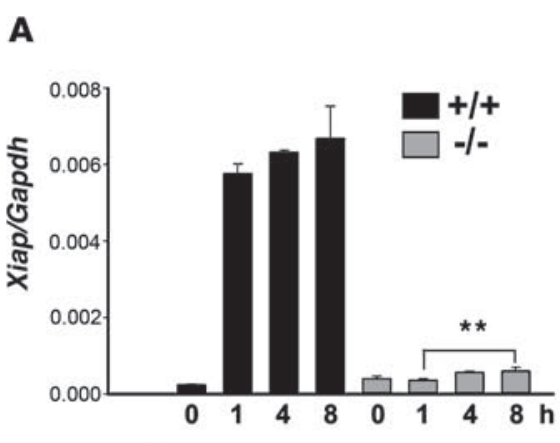

B
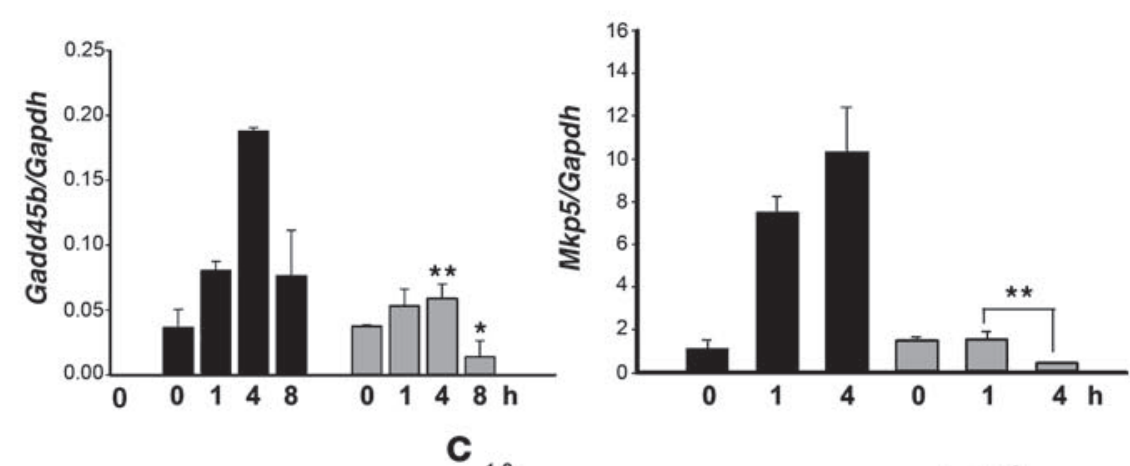

C

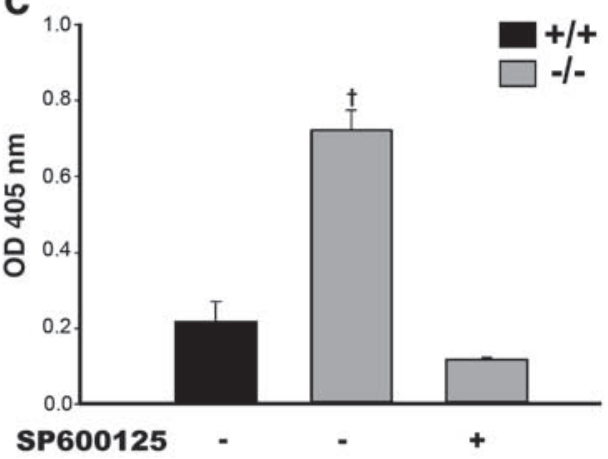

D

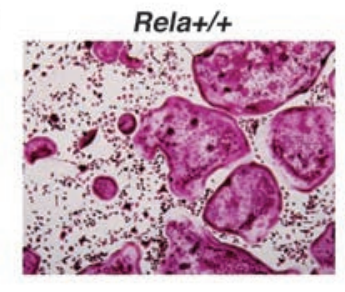

Rela-/-

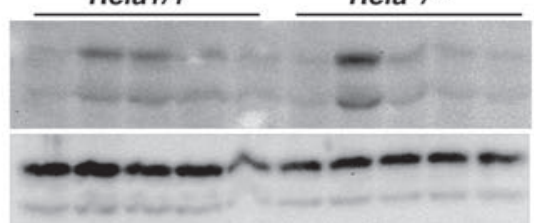

RANKL $015^{\prime} 30^{\prime}$ ' $1 \mathrm{~h}$ 3h 0 15' $30^{\prime}$ ' $1 \mathrm{~h}$ $3 \mathrm{~h}$
pJNK

JNK

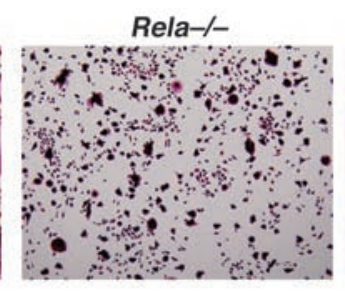

Rela-1- + SP600125

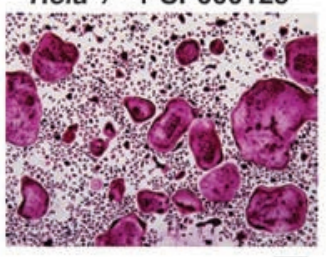

Figure 4

The absence of RelA leads to enhanced JNK activation. (A) Pre-OCs (BMMs cultured for 2 days with M-CSF and RANKL) generated from $\mathrm{Rela}^{+/+}$(black bars) and Rela-/- (gray bars) mice were then starved of serum and cytokines and restimulated with RANKL for the indicated times prior to RNA extraction and real-time quantitative PCR for xiap, Gadd45b, and Mkp5. Rela ${ }^{-1}$ pre-OCs show significantly less induction of xiap, Gadd45b and Mkp5. ${ }^{*} P<0.5,{ }^{* *} P<0.01$ compared to Rela ${ }^{+/+}$at the same time point. (B) $R e / a^{+/+}$and Rela ${ }^{-/}$pre-OCs were starved and restimulated with RANKL for the indicated times, and total lysates were analyzed by immunoblot for pJNK, demonstrating enhanced JNK activation in Rela ${ }^{-/}$cultures. A parallel blot with equal loading was used to assess total JNK protein. (C) Rela ${ }^{+/}$and Rela ${ }^{-/-}$BMMs were cultured in RANKL, with or without the JNK inhibitor SP600125 $(1 \mu \mathrm{m})$, for 36 hours Apoptosis was then assessed by DNA fragmentation assay. RANKL-mediated apoptosis was abrogated by the JNK inhibitor in Rela ${ }^{-/}$cells. The data are representative of 3 independent experiments. ${ }^{\dagger} P<0.05$ Rela $^{-/-}$versus $\mathrm{Rela}^{+/+}$or Rela ${ }^{-/-}$with SP600125. (D) Rela ${ }^{+/+}$and Rela ${ }^{-/-}$BMMs were cultured in osteoclastogenic conditions for 8 days, with or without SP600125 for the first 36 hours, showing rescue of differentiation with short-term JNK inhibition. Scale bar: $200 \mu \mathrm{m}$.

transduced with retroviral siRNA hairpins were decreased $85 \%$ for siBid 1 and $55 \%$ for siBid 2 compared with those transduced with an irrelevant siRNA-targeting luciferase, siLuc (Figure 5B). Culture of the same set of BMMs in RANKL showed a reduction of apoptosis back to Rela ${ }^{+/+}$levels (Figure 5C) and restored OC numbers after 6 days of culture (Figure 5D). Furthermore, analysis of bone resorption by OCs derived from siBid-transduced BMMs demonstrated that the function of surviving OCs was intact (Figure 5, E and F). The degree of Bid knockdown correlated with rescue of apoptosis, differentiation, and function. Thus, RelA opposes a proapoptotic pathway mediated by JNK and Bid in OC precursors.

Protection from RANKL-induced apoptosis is specific for RelA and not RelB. Previous studies of the function of NF- $\mathrm{KB}$ in OCs, including our own study of $\operatorname{NIK}(8,9)$, have not established unique roles for individual subunits. Therefore, we examined the effect of RelA on the $\kappa \mathrm{B}$ DNA-binding activity of RelB, controlled by the alternative

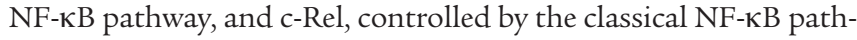
way. In RANKL-stimulated BMMs, levels of RelB $\kappa B$-binding activity are similar in the presence and absence of RelA (Figure 6A), indicating that activation of the alternative pathway is not affected in these mice. The $\kappa \mathrm{B}$ DNA-binding activity of $\mathrm{c}$-Rel is also unchanged in Rela $^{-/-}$cultures compared with Rela ${ }^{+/+}$cultures, suggesting that c-Rel does not perform the same function as RelA.

To further address whether the classical subunit RelA has a distinct function from the alternative NF- $\kappa B$ subunit RelB, we analyzed apoptosis in RelB-deficient cultures. In contrast to Rela-/- BMMs, Relb ${ }^{-/}$ BMMs did not undergo apoptosis when cultured for 48 hours in the presence of RANKL (Figure 6B). Next, we asked if the overexpression of RelB could rescue the apoptosis induced by RANKL in RelA-deficient OC precursors by using retroviral vectors to transduce Rela-/BMMs. Immunoblots confirmed retroviral-mediated expression of both subunits (Figure 6C). As expected, expression of RelA reduced 

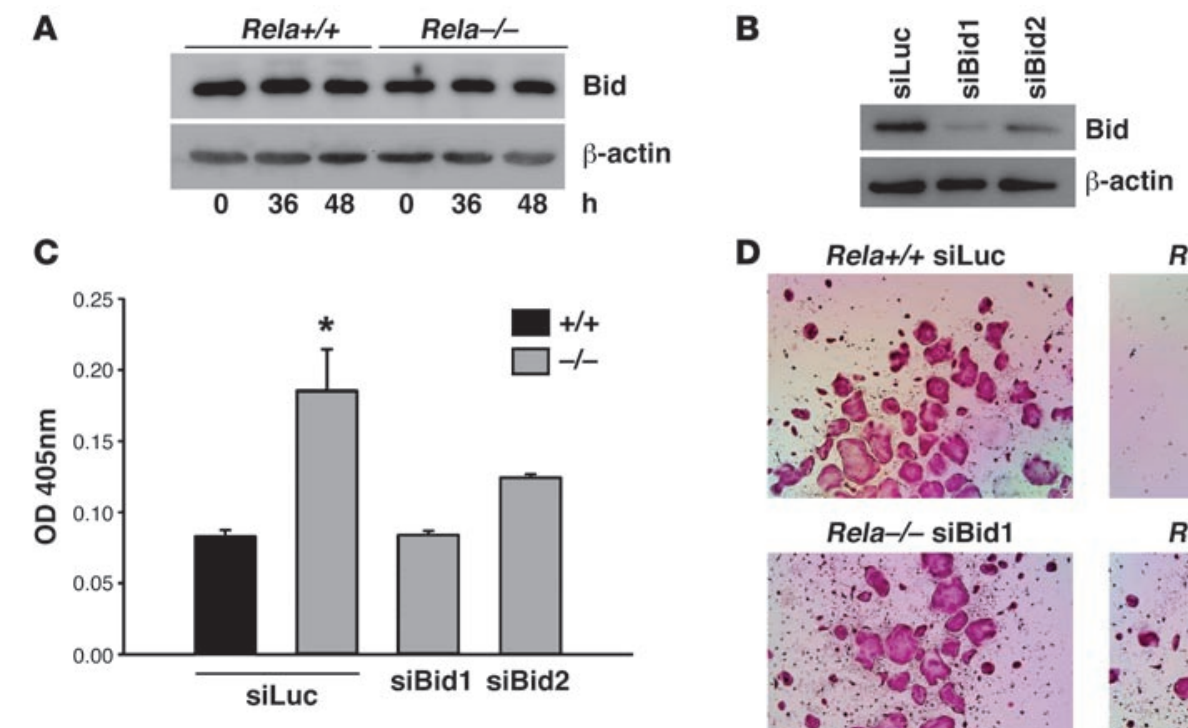

D

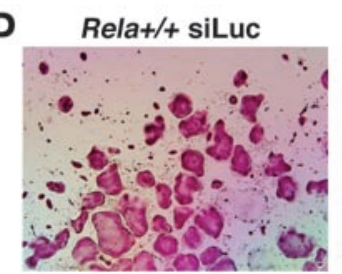

Rela-/-siBid1

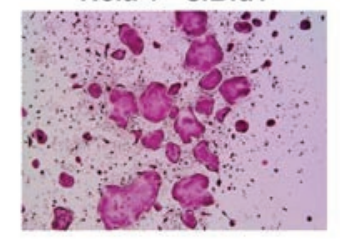

F

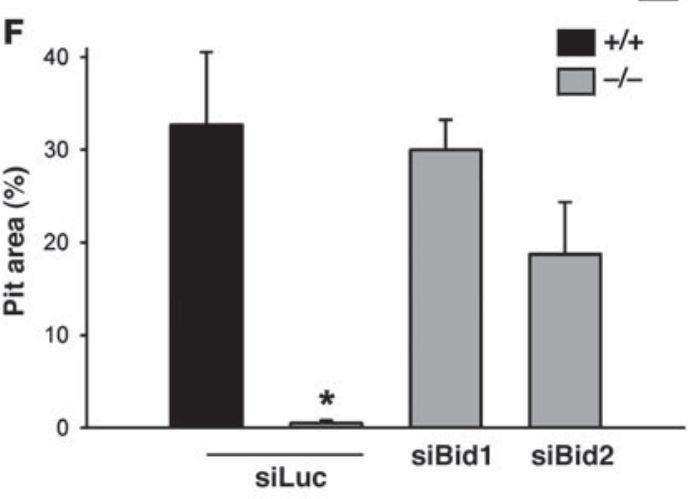

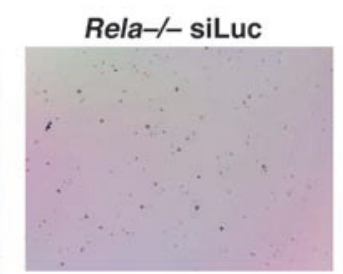

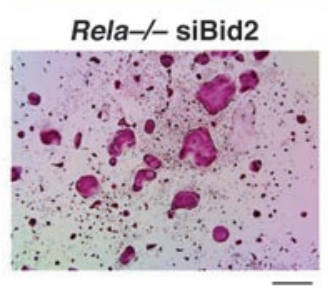$$
\text { . }
$$
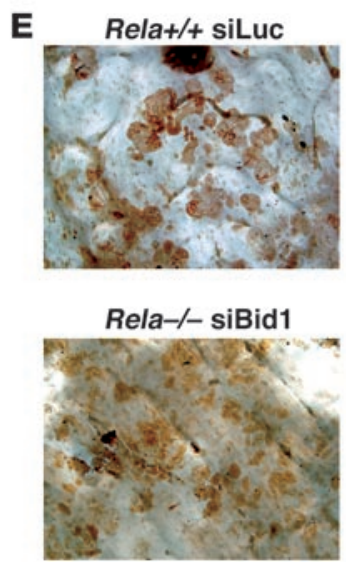

Rela-/-siLuc

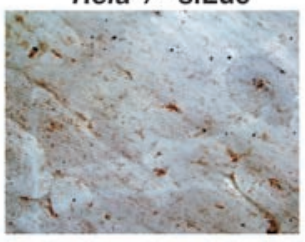

Rela-/-siBid2

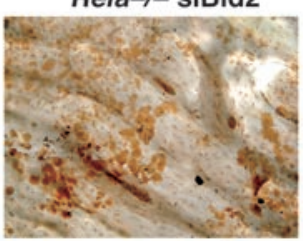


A

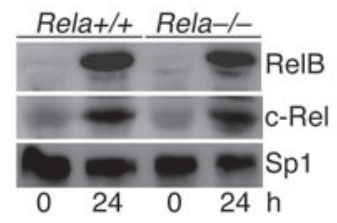

\section{C}
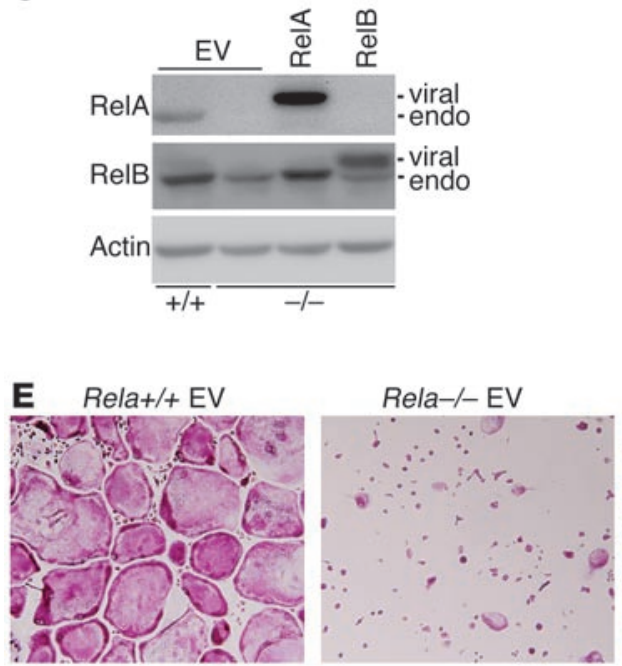

Rela-l-EV
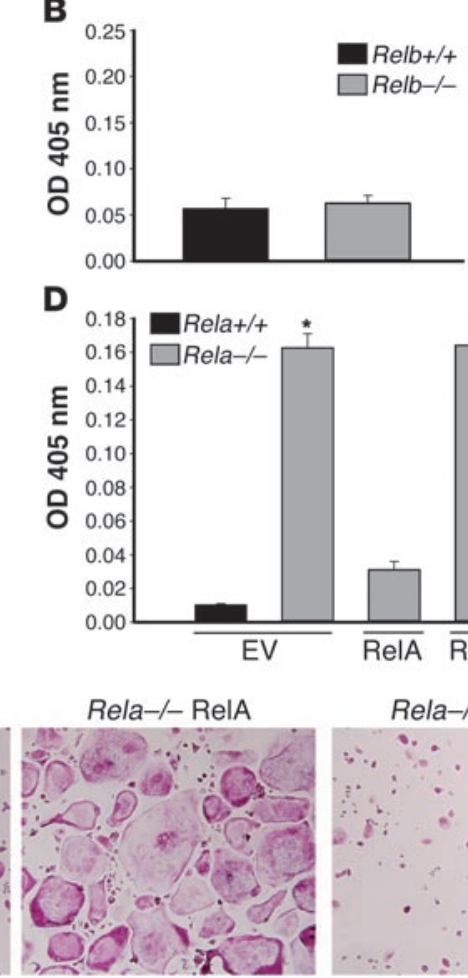

D

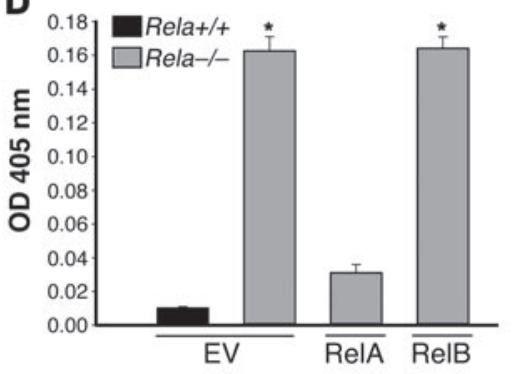

Figure 6

Enhanced RANKL-induced apoptosis is specific for RelA. (A) BMMs from Rela ${ }^{+/+}$and Rela Re $^{-/-}$mice were cultured in the presence of M-CSF and RANKL for 0 or 24 hours. Equal amounts of nuclear extracts were incubated with biotinylated $\kappa B$ oligos bound to streptavidin-coated beads. Beads were washed to isolate $\kappa \mathrm{B}$-bound proteins, and these were analyzed by immunoblot for RelB or c-Rel. Unbound nuclear proteins were analyzed by immunoblot for Sp1 as loading control. (B) BMMs from Rel/ $\mathrm{b}^{+/+}$and $R_{\text {ell }} /-$ mice were cultured with M-CSF and RANKL for 48 hours, and apoptosis was assessed by DNA fragmentation assay, showing no effect of RelB on apoptosis. (C) Rela ${ }^{-/}$BMMs retrovirally transduced with empty vector (EV), RelA, or RelB, and Rela+/+ BMMs transduced with EV were selected in blastocydin, then analyzed by immunoblot. Viral-encoded proteins (both RelA and RelB), migrate more slowly than their endogenous (endo) counterparts, and are expressed at 2- to 3-fold over endogenous levels. (D) BMMs transduced in C were cultured with RANKL for 48 hours, and apoptosis was assessed by DNA fragmentation assay. While the EV control and RelB vectors do not abrogate the RANKL-induced cell death in Rela ${ }^{-/}$cultures, RelA reduces apoptosis to the same level as Rela ${ }^{+/+}$transduced with EV. ${ }^{*} P<0.001$ Rela ${ }^{+/+}$EV. (E) Transduced BMMs were grown in osteoclastogenic conditions for 6 days and then were fixed and stained for TRAP. Reexpression of RelA restores Rela ${ }^{-/}$OC differentiation, while overexpression of RelB has no effect. Scale bar: $200 \mu \mathrm{m}$.

DNA binding of RelA-containing dimers. Blockade of this classical pathway, by mutation or inhibition of IKK $\beta$ or IKB-superrepressors, prevents activation of RelA and blocks osteoclastogenesis (5, $7,14)$. It therefore appeared that RelA was an important mediator of OC differentiation. We found that although Rela ${ }^{-/}$precursors do not differentiate well in standard osteoclastogenic culture conditions, this effect is completely dependent on an increase in apoptosis. When cell death is blocked, differentiation proceeds and fully functional bone-resorbing OCs are generated. We therefore conclude that RelA is not specifically required for the OC differentiation transcriptional program.

TNFR1 bears a death domain in its cytoplasmic tail and can cause apoptosis via caspase-8. The role of NF-KB in opposing this apoptotic TNF pathway has been studied extensively in several systems, and transcriptional targets have been described. In the OC lineage, blockade of classical NF-KB leads to TNF-mediated apoptosis $(5,6)$. In con-

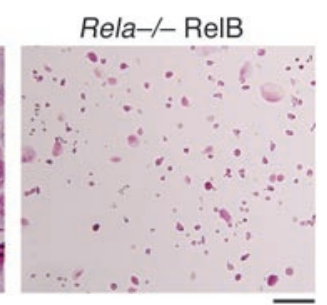

trast to TNFR1, RANK does not have a death domain, and therefore, lacks an obvious mechanism by which to induce apoptosis. Nevertheless, at least 1 study using the RAW cell line (13) has suggested that RANKL can induce apoptosis in OC precursors dependent on JNK but independent of classical NF-кB. We find that in primary marrow macrophages lacking both RelA and TNFR1, RANKL is a potent inducer of apoptosis mediated by JNK, Bid, and caspase-3. The effect is dependent on the loss of RelA and not TNFR1, since the phenotype is not changed by reexpression of TNFR1. Furthermore, Li et al. (22) recently examined the bone phenotype of TNFR1-deficient mice and found elevated bone formation, due to increased osteoblast activity, but no change in bone resorption or OC parameters. These data support our conclusion that RANKL alone is able to induce apoptosis in OC precursors in the absence RelA, independent of TNF effects. Additionally, because TNF can cause apoptosis via TNFR1 in RelA-deficient cells (23), we would predict that the absence of TNFR1 would be more likely to reduce the apoptotic response (as occurs in hepatocytes), rather than potentiate it.

The mechanism by which JNK activation leads to cell death has only been studied in the context of TNF signaling. Franzoso and colleagues $(18,24)$ have shown that RelA mediates transcription of Gadd $45 \beta$, an inhibitor of MKK7, the activating kinase for JNK. In their model, absence of RelA downregulates Gadd $45 \beta$, leading to prolonged activation of MKK7 and JNK in response to TNF. This aberrantly activated JNK causes cleavage of the proapoptotic Bcl-2 family member Bid to an active jBid form, leading to its translocation to the mitochondria and activation of the caspase-3 pathway (25). We find that RANKL fails to upregulate Gadd45 $\beta$, as well as MKP5, in the absence of RelA and that activation of JNK is enhanced. Short-term blockade of JNK activation prevents RANKL-induced apoptosis in OC precursors, allowing their differentiation, as does reduction of Bid levels by siRNA. However, we are unable to detect the appearance of cleaved forms of Bid in our Rela ${ }^{-1}$ OC precursors, suggesting that there may be some differences in the pathways due to the cell lineage or the receptor initiating the pathway. In the context of anoikis (cell death induced by lack of adhesion), Bid can be activated without cleavage (26).

In the TNF pathway, ROS have been identified as the key mediator of the crosstalk between the NF-KB and JNK (27). In situations of impaired NF- $\mathrm{KB}$ signaling, generation of ROS is required for 
TNF-mediated apoptosis. In the OC lineage, however, inhibition of ROS reduces differentiation and bone resorption $(28,29)$. JNK activation is also required for OC differentiation (30-32). RANKL activates both ROS and JNK, and these linked pathways contribute both differentiation and apoptotic signals. In the presence of RelA, apoptosis is suppressed and generation of OCs is optimal, while in the absence of RelA, precursor apoptosis reduces the number of mature bone-resorbing cells. Thus, there is a tight range of JNK activation required for osteoclastogenesis, and RelA is a critical component in maintaining appropriate levels. Furthermore, inhibition of caspases or JNK within the first 2 days of RANKL culture restores differentiation in $\mathrm{Rela}^{-/-}$OC precursors, suggesting that timing of JNK activation might be as important as the magnitude.

Karin and coauthors (33) showed that TNF- $\alpha$-mediated JNK activation induces apoptosis by accelerating turnover of the NF- $\mathrm{BB}$ regulated protein c-FLIP, an inhibitor of caspase-8, by upregulating the E3 ubiquitin ligase Itch. However, in Rela ${ }^{-/}$OC precursors RANKL does not induce caspase- 8 activation, so it is unlikely that c-FLIP and Itch are important mediators in response to RANKL.

RelA and RelB appear to have distinct functions in the OC lineage. In the absence of RelA, OC precursors readily undergo apoptosis in response to RANKL but are capable of full differentiation and function if cell death is blocked. In contrast, RelB-deficient precursors are not susceptible to RANKL-mediated apoptosis but have a defect in differentiation (21). Absence of RelA does not affect signaling by RelB, and overexpression of the latter does not rescue the apoptotic Rela/- phenotype. Another major difference between RelA and RelB is the kinetics of their activation by RANKL. RelA is activated within minutes of exposure of BMMs to RANKL and is relatively transient, while RelB activation takes hours and persists for days (D.V. Novack, unpublished observations). Whether the differences in RelA and RelB function lie in their upstream control mechanisms (IKK $\beta$ and NIK, respectively) or in the structure of the subunits themselves remains to be determined.

Previous studies have shown that inhibition of the classical NF- $\kappa \mathrm{B}$ pathway blocks both inflammation and bone erosion in several models of inflammatory arthritis. We have now used radiation chimeras bearing bone marrow from Rela $^{-/-}$Tnfr $1^{-/-}$mice or from Rela ${ }^{+/}$Tnfr1 $1^{-/-}$controls in the serum transfer model of arthritis. Despite the fact that hematopoietic cells in these RCs are unable to respond directly to TNF, inflammation develops with or without RelA. However, the presence of RelA was critical for the OC response. The number of OCs adjacent to inflammatory pannus on bone surfaces and their activity as measured by serum CTX levels were both markedly blunted in $\mathrm{Rela}^{-/-} \mathrm{RCs}$. This effect is most likely mediated by RANKL, as opposed to other cytokines present during arthritis, since Rela-/- RCs also have a poor osteoclastogenic response to injection of exogenous RANKL.

In summary, we have demonstrated that despite the absence of a cytoplasmic death domain, RANK activates a proapoptotic pathway similar to that induced by TNFR1, with JNK, Bid, and caspase-3 playing key roles. The NF-кB subunit RelA has a central, subunit-specific function in blocking this cell death to allow differentiation but is not required for the transcription of genes required for osteoclastogenesis.

\section{Methods}

Mice. Rela ${ }^{-/-} \mathrm{Tnfr}^{-/-}$and $\mathrm{relb}^{-/-}$mice were maintained by heterozygote matings in a specific pathogen-free facility. The genotype of all mice used was confirmed by PCR analysis of genomic DNA extracted from tail biopsies.
All experimental protocols were approved by the Institutional Animal Studies Committee at Washington University School of Medicine.

Bone marrow transplantation. Recipient 3- to 5-week-old C57BL/6J mice (The Jackson Laboratory) were administered 10 Gy total-body $\gamma$-irradiation 1 day prior to injection of $1 \times 10^{6}$ bone marrow cells from Rela $^{+/}$Tnfr1 $1^{-/-}$or Rela ${ }^{-/}$Tnfri $1^{-/-}$donors via tail vein. The engraftment of transplanted marrow was confirmed by western blot for RelA (data not shown) on BMMs from the RCs.

Serum transfer arthritis. Arthritis was induced by intraperitoneal injection of $250 \mu \mathrm{K} / \mathrm{BxN}$ serum at days 0, 2, and 7, with $50 \mu \mathrm{g}$ LPS (E. coli 011; B4; Sigma-Aldrich) on day 2 as described (9). Ankle thickness in the hind paws was measured by dial gauge (Mitutoyo).

In vivo RANKL treatment. Rela ${ }^{+/+}$and Rela-/- RCs were injected subcutaneously with GST-RANKL (500 $\mu$ g per day for 5 days) or PBS (sham).

Measurement of serum TRAP5b and CTX. Immediately prior to sacrifice, mice were anesthetized and bled retro-orbitally. After clotting and centrifugation, serum was collected and stored at $-80^{\circ} \mathrm{C}$ prior to analysis using Mouse TRAP assay (IDS Inc.) or CTX (Rat Lap Elisa; Nordic Bioscience Diagnostic) according to the manufacturer's instructions.

Bone histomorphometry. Mouse lumbar vertebrae, calvariae, or ankle joints were fixed overnight in formalin and decalcified in 14\% EDTA. Paraffinembedded sections were stained with H\&E or for TRAP activity. Bone sections were coded prior to analysis. Trabecular bone volume (BV/TV), OC surface (Oc.S/BS.), and OC numbers (N.Oc./B.Pm.) were measured by an observer blinded to genotype according to a standard protocol using Bioquant Osteo (Bioquant Image Analysis Corporation).

OC culture. BMMs were obtained and differentiated into OCs as described (34), using CMG14-12 as a source of M-CSF and recombinant GSTRANKL. For osteoclastogenesis in 96-well plates, BMMs from Rela-/- and Rela $^{+/+}$mice were plated at $5 \times 10^{3}$ and cultured with $30 \mathrm{ng} / \mathrm{ml}$ of M-CSF (1:50 CMG supernatant). After 5 to 6 days the wells were fixed with $4 \%$ paraformaldehyde and OCs were visualized by histochemical staining for TRAP activity according to manufacturer's instructions (Sigma-Aldrich). For coculture experiments, calvarial OCs and BMMs from $\mathrm{Rela}^{-/}$and $\mathrm{Rela}^{+/+}$ mice were mixed at a ratio of $1: 2$ and cultured for 7 days in the presence of 1,25 vitamin $\mathrm{D}_{3}\left(10^{-8} \mathrm{M}\right)$ (Sigma-Aldrich).

Bone pit staining. BMMs were differentiated on bovine cortical bone slices with RANKL for 7-8 days. The staining of resorption lacuna was performed as described previously (35). Briefly, the cells were removed from the bone slices by wiping the surface of the bone slices with a soft brush, then slices were rinsed, incubated with $20 \mu \mathrm{g} / \mathrm{ml}$ peroxidase-conjugated wheat germ agglutinin (Sigma-Aldrich) for 30 minutes, and developed with 3, 3'-diaminobenzidine for 15 minutes.

MTT assay. BMMs were cultured in M-CSF, with or without GST-RANKL, for 3 to 48 hours, and the number of viable cells was measured by adding $10 \mu \mathrm{l}$ MTT $5 \mathrm{mg} / \mathrm{ml} \mathrm{(3-4,} \mathrm{5-dimethylthiazol-2y-2,5} \mathrm{diphenyltetrazolium} \mathrm{bro-}$ mide; Sigma-Aldrich) to $200 \mu \mathrm{l}$ culture medium in each well and incubated at $37^{\circ} \mathrm{C}$ for 4 hours. To stop the reaction, $150 \mu \mathrm{l} 0.04 \mathrm{~N} \mathrm{HCl}$ in isopropanol was added to each well, and absorbance was determined at $570 \mathrm{~nm}$.

DNA fragmentation assay. Cell death detection assay was performed with the Cell Death Detection Elisa kit (Roche Diagnostics Corp.) according to manufacturer's instructions. Rela ${ }^{+/+}$and Rela ${ }^{-/-}$BMMs were cultured in 96-well plates for 36-48 hours in the presence of M-CSF (1:50 CMG supernatant) and GST-RANKL $(150 \mathrm{ng} / \mathrm{ml})$, with or without $10 \mu \mathrm{M}$ ZVAD-FMK (R\&D Systems) or the JNK inhibitor, SP600125 $(1.0 \mu \mathrm{M})$ (Calbiochem) prior to lysis. Absorbance was read at $405 \mathrm{~nm}$ using Bio-Rad Microplate Reader.

Caspase activation fluorometric assays. The activation of caspase- $3,-8$, or -9 was measured according to the protocol of their respective fluorometric kits (Biovision). Cells in suspension were collected by centrifugation and mixed with adherent cells for lysis. Substrates were DEVD-AFC (cas- 
pase-3), FLICE (caspase-8), and LEHD-AFC (caspase-9). Fluorescence was read using Spectra Fluor (Tecan) with 400-nm excitation filter and 505-nm emission filter.

Retroviral transduction. Retrovirus was generated by cloning murine relA (with an N-terminal HA tag) or relB cDNAs into the pMX-IRES-bsr vector adapted for the Gateway cloning system (Invitrogen) and transfected into platE packaging cells, using Transfectol (Gene Choice). Replication incompetent viral supernatant was added to BMMs for 24 hours, along with $4 \mu \mathrm{g} / \mathrm{ml}$ polybrene (Sigma-Aldrich), prior to selection with blastocydin $(1 \mu \mathrm{g} / \mathrm{ml})$ for 3 days. Surviving, transduced BMMs were then cultured in a similar fashion to primary, unmanipulated BMMs. siRNAs for Bid were designed using the Dharmacon website (http://www.dharmacon.com/ DesignCenter) and cloned as a small hairpin into pSuper retroviral vector bearing the U6 promoter. SiBid 1 sequence derived from the 3 ' untranslated region is $5^{\prime}$-CAGCTTGAGTGTATCTGAA-3'; SiBid2 sequence derived from the open reading frame is $5^{\prime}$-GCTCTGGCTGTACTCGCCA- 3 '. PlatE packaging cells were transfected and BMMs were transduced as for the PMX vectors but with puromycin $(2 \mu \mathrm{g} / \mathrm{ml})$ selection, and the expression levels for BID were analyzed by western blot.

Immunoblotting analysis. Total protein was extracted as described (8). The proteins were quantitated with DC protein assay kit (Bio-Rad), and 40-50 $\mu \mathrm{g}$ of total protein were resolved by SDS-polyacrilamide gel electrophoresis under reducing conditions and then transferred to PVDF membrane. The blots were incubated for 1 hour in 5\% dry milk and subsequently, overnight with the specific antibodies for pJNK, total JNK (Cell Signalling); RelA, RelB, c-Rel, SP1, $\beta$-actin (Santa Cruz Biotechnology Inc.); or Bid (R\&D Systems); followed by anti-rabbit or anti-mouse HRP-conjugated secondary antibodies (Santa Cruz Biotechnology Inc.). Blots were evaluated by chemiluminescence using SuperSignal substrates (Pierce) and imaged with Genesnap, using the Syngene Imaging System (Synoptics). Relative protein expression was determined using Genetools (Synoptics).

$\kappa B$ pulldown assay. Nuclear extract $(30 \mu \mathrm{g})$ was incubated with streptavidin-coated agarose beads preincubated with biotinylated $\kappa B 3$ oligonucleotide (8) for 30 minutes at $4{ }^{\circ} \mathrm{C}$ on a rotator in $1 \mathrm{x}$ binding buffer (30 mM NaCl, 10 mM Tris, pH 7.4, 1 mM EDTA, 5\% glycerol, $1 \mathrm{mg} / \mathrm{ml}$ BSA, and $1 \mathrm{mM}$ DTT) with $1 \mu \mathrm{g}$ poly dIdC. Beads were then washed in $1 \mathrm{x}$ binding buffer 3 times prior to SDS-PAGE and immunoblotted for RelB and/or c-Rel.
Real-time PCR. Total RNA was extracted using RNeasy (QIAGEN), and CDNA was generated with AMV-RT (Fisher) according to manufacturers' instructions using $1 \mu \mathrm{g}$ total RNA and oligo-dT primers diluted to a final volume of $100 \mu \mathrm{l}$. Quantitative RT-PCR was performed on an ABI7300 Real-Time PCR system (Applied Biosystems) using SYBR Green PCR Master Mix assay (Applied Biosystems). The standard curve used a series of duplicate dilutions of plasmid for each gene and GAPDH cDNA. The amplification reaction was performed for 40 cycles with denaturation at $95^{\circ} \mathrm{C}$ for 10 minutes, followed by annealing at $95^{\circ} \mathrm{C}$ for 15 seconds, and extension and detection at $60^{\circ} \mathrm{C}$ for 1 minutes. The relative RNA abundance of each target gene transcript was normalized against endogenous gene control and calculated as $2^{-\Delta c t}$ (target gene - endogenous control gene), where $c t$ represents the threshold cycle for each transcript.

Primers are as follows: Gapdh, 5'-CTTCACCACCATGGAGAAGGC-3' and 5'-GACGGACACATTGGGGGTAG-3'; Xiap, 5'-TGCAAGAGCTGGATTTTATGC-3' and 5'-GGTCTTCACTTGGCTTCCAAT-3'; Gadd45b, 5'-TACGAGGCGGCCAAACTGATGAAT-3' and 5'-ACGACTGGATCAGGGTGAAGTGAA-3'; Mkp5, 5'-GAGAAAGGCCTCTTCAACTACAA$3^{\prime}$ and $5^{\prime}$-CCACACTGGTGAGCTTCCTC-3'.

Statistics. Data are presented as mean \pm SEM. Statistical significance was determined by 2 -tailed Student's $t$ test using SigmaPlot (SSPS). $P$ values $\leq 0.05$ were considered significant.

\section{Acknowledgments}

We thank D. Baltimore (California Institute of Technology, Pasadena, California, USA) for Rela ${ }^{-1}$ mice and Crystal Idleberg for expert animal histology. This work was supported by grants from the National Institutes of Health AR47846, CA103035, and AR52705 (to D.V. Novack); and AR52921 (to R. Faccio). Additional support was from the Arthritis Foundation (to R. Faccio).

Received for publication July 25, 2007, and accepted in revised form March 26, 2008.

Address correspondence to: Deborah Veis Novack, Washington University School of Medicine, 660 S. Euclid Ave., Box 8301, St. Louis, Missouri 63110, USA. Phone: (314) 454-8472; Fax: (314) 454-5047; E-mail: novack@wustl.edu.
1. Teitelbaum, S.L. 2007. Osteoclasts: what do they do and how do they do it? Am. J. Pathol. 170:427-435.

2. Perkins, N.D. 2007. Integrating cell-signalling pathways with NF-KB and IKK function. Nat. Rev. Mol. Cell Biol. 8:49-62.

3. Franzoso, G., et al. 1997. Requirement for NF-кB in osteoclast and B-cell development. Genes Dev. 11:3482-3496

4. Iotsova, V., et al. 1997. Osteopetrosis in mice lacking NF-кB1 and NF-кB2. Nat. Med. 3:1285-1289.

5. Ruocco, M.G., et al. 2005. IкB kinase (IKK) $\beta$, but not IKK $\alpha$, is a critical mediator of osteoclast survival and is required for inflammation-induced bone loss. J. Exp. Med. 201:1677-1687.

6. Abbas, S., and Abu-Amer, Y. 2003. Dominant-negative IKB facilitates apoptosis of osteoclasts by tumor necrosis factor- $\alpha$. J. Biol. Chem. 278:20077-20082.

7. Abu-Amer, Y., Dowdy, S.F., Ross, F.P., Clohisy, J.C., and Teitelbaum, S.L. 2001. TAT fusion proteins containing tyrosine 42-deleted I $\mathrm{B} \mathrm{B} \alpha$ arrest osteoclastogenesis. J. Biol. Chem. 276:30499-30503.

8. Novack, D.V., et al. 2003. The IкB function of NF$\kappa \mathrm{B} 2$ p100 controls stimulated osteoclastogenesis. J. Exp. Med. 198:771-781.

9. Aya, K., et al. 2005. NF-кB-inducing kinase controls lymphocyte and osteoclast activities in inflammatory arthritis. J. Clin. Invest. 115:1848-1854.
10. Beg, A.A., Sha, W.C., Bronson, R.T., Ghosh, S., and Baltimore, D. 1995. Embryonic lethality and liver degeneration in mice lacking the RelA component of NF-kappa B. Nature. 376:167-170.

11. Beg, A.A., and Baltimore, D. 1996. An essential role for NF-kappaB in preventing TNF-alpha-induced cell death. Science. 274:782-784.

12. Van Antwerp, D.J., Martin, S.J., Kafri, T., Green, D.R., and Verma, I.M. 1996. Suppression of TNF- $\alpha$ induced apoptosis by NF-кB. Science. 274:787-789.

13. Bharti, A.C., Takada, Y., Shishodia, S., and Aggarwal, B.B. 2004. Evidence that receptor activator of nuclear factor (NF)- $\kappa \mathrm{B}$ ligand can suppress cell proliferation and induce apoptosis through activation of a NF-KB-independent and TRAF6-dependent mechanism. J. Biol. Chem. 279:6065-6076.

14. Jimi, E., et al. 2004. Selective inhibition of NF-кB blocks osteoclastogenesis and prevents inflammatory bone destruction in vivo. Nat. Med. 10:617-624.

15. Clohisy, J.C., et al. 2003. Direct inhibition of NF-KB blocks bone erosion associated with inflammatory arthritis. J. Immunol. 171:5547-5553.

16. Korganow, A., et al. 1999. From systemic T cell selfreactivity to organ-specific autoimmune disease via immunoglobulins. Immunity. 10:451-461.

17. Salvesen, G.S., and Duckett, C.S. 2002. IAP proteins: blocking the road to death's door. Nat. Rev.
Mol. Cell Biol. 3:401-410

18. De Smaele, E., et al. 2001. Induction of gadd $45 \beta$ by NF-KB downregulates pro-apoptotic JNK signalling. Nature. 414:308-313.

19. Kamata, H., et al. 2005. Reactive oxygen species promote TNFalpha-induced death and sustained JNK activation by inhibiting MAP kinase phosphatases. Cell. 120:649-661.

20. Papa, S., Zazzeroni, F., Pham, C.G., Bubici, C., and Franzoso, G. 2004. Linking JNK signaling to NF-кB: a key to survival. J. Cell Sci. 117:5197-5208.

21. Vaira, S., et al. 2008. RelB is the NF-KB subunit downstream of NIK responsible for osteoclast differentiation. Proc. Natl. Acad. Sci. U. S. A. 105:3897-3902.

22. Li, Y., et al. 2007. Endogenous TNF $\alpha$ lowers maximum peak bone mass and inhibits osteoblastic Smad activation through NF-кB. J. Bone Miner. Res. 22:646-655.

23. Ruocco, M.G., et al. 2005. IкB kinase (IKK) $\beta$, but not IKK $\alpha$, is a critical mediator of osteoclast survival and is required for inflammation-induced bone loss. J. Exp. Med. 201:1677-1687.

24. Papa, S., et al. 2007. Insights into the structural basis of the GADD45beta-mediated inactivation of the JNK kinase, MKK7/JNKK2. J. Biol. Chem. 282:19029-19041.

25. Deng, Y., Ren, X., Yang, L., Lin, Y., and Wu, X. 2003. 
A JNK-dependent pathway is required for TNF $\alpha$ induced apoptosis. Cell. 115:61-70.

26. Valentijn, A.J., and Gilmore, A.P. 2004. Translocation of full-length Bid to mitochondria during anoikis. J. Biol. Chem. 279:32848-32857.

27. Papa, S., et al. 2006. The NF-kB-mediated control of the JNK cascade in the antagonism of programmed cell death in health and disease. Cell Death Differ. 13:712-729.

28. Lee, N.K., et al. 2005. A crucial role for reactive oxygen species in RANKL-induced osteoclast differentiation. Blood. 106:852-859.

29. Kim, H.J., et al. 2006. Antioxidant $\alpha$-lipoic acid inhibits osteoclast differentiation by reducing nuclear factor- $\mathrm{KB}$ DNA binding and prevents in vivo bone resorption induced by receptor activator of nuclear factor- $\mathrm{B}$ ligand and tumor necrosis factor- $\alpha$. Free Radic. Biol. Med. 40:1483-1493.

30. Yamanaka, Y., Abu-Amer, Y., Faccio, R., and Clohisy, J.C. 2006. Map kinase c-JUN N-terminal kinase mediates PMMA induction of osteoclasts. J. Orthop. Res. 24:1349-1357.

31. David, J.-P., Sabapathy, K., Hoffmann, O., Idarraga, M.H., and Wagner, E.F. 2002. JNK1 modulates osteoclastogenesis through both c-Jun phosphorylation-dependent and -independent mechanisms. J. Cell Sci. 115:4317-4325.

32. Lee, S.K., Gardner, A.E., Kalinowski, J.F., Jastrzebski, S.L., and Lorenzo, J.A. 2006. RANKL-stimulated osteoclast-like cell formation in vitro is partially dependent on endogenous interleukin-1 production. Bone. 38:678-685.

33. Chang, L., et al. 2006. The E3 ubiquitin ligase itch couples JNK activation to TNFalpha-induced cell death by inducing c-FLIP(L) turnover. Cell. 124:601-613.

34. Faccio, R., Novack, D.V., Zallone, A., Ross, F.P., and Teitelbaum, S.L. 2003. Dynamic changes in the osteoclast cytoskeleton in response to growth factors and cell attachment are controlled by $\beta 3$ integrin. J. Cell Biol. 162:499-509.

35. Zhao, H., Laitala-Leinonen, T., Parikka, V., and Vaananen, H.K. 2001. Downregulation of small GTPase Rab7 impairs osteoclast polarization and bone resorption. J. Biol. Chem. 276:39295-39302. 